\title{
Chiral restoration and the extended photoabsorption sum rule in nuclei
}

\author{
M. Ericson \\ Institut de Physique Nucléaire de Lyon, IN2P3-CNRS \\ and Université Claude Bernard \\ and CERN CH-1211 Geneva 23 \\ M. Rosa-Clot \\ Department of Physics, University of Florence, and INFN Florence Italy \\ S.A. Kulagin \\ Institute for Nuclear Research of The Russian Academy of Science, \\ 60-th October Anniversary Pr. 7a, Moscow 117312, Russia
}

\begin{abstract}
The Bethe-Levinger sum rule is extended beyond the potential model. The pion degrees of freedom are taken into account and the modifications of the potential theory are analysed within two different approaches: dipole sum rule and dispersion relation on the Compton amplitude. Our aim is to extract from the photon data experimental information on the expectation value of the square of the pion field, a quantity which enters also in the restoration of chiral symmetry in nuclei and in pion-nucleus scattering. We are led to incorporate in the description the $\Delta$ resonance, which is strongly excited by the pion degrees of freedom.
\end{abstract}

Typeset using REVTEX 


\section{INTRODUCTION}

The forward Compton amplitude is sensitive to the pionic structure of nuclei, through its link [1] to the enhancement factor $K$ of the dipole sum rule [2]. In the original derivation of the sum rule the enhancement arises mostly from the tensor correlations, i.e. from the pion. It is thus natural to investigate how the explicit introduction in the electromagnetic current of the pion degree of freedom affect the sum rule, especially in view of the fact that the pion has a small mass, which is not far from the nuclear excitation domain. This is known to create difficulties in the actual evaluation of $K$ as it is not possible to disentangle the nuclear excitations in the photoproduction region. We have shown in a previous work [3] that the usual enhancement factor provides information on the tensor part of the spinisospin correlations. By extending the sum rule to incorporate the pion degrees of freedom it is possible to explore the expectation value of the squared pion field, a quantity that enters in a number of other problems such as the restoration of chiral symmetry in nuclei and in pion-nucleus scattering. This is the aim of this work.

The explicit inclusion of pions in the electromagnetic current entering in the dipole

sum rule has been previously performed by Noble [4], who found a strong reduction of enhancement factor ( $K \approx 0.2$ instead of $K \approx 1$ ). In the present work, we will show that this is because the inclusion of pions has an effect not only on the enhancement factor, but also on the integral over the cross-section, since the photoproduction region has to be incorporated. This guarantees the consistency of the new sum rule, which defines the enhancement factor.

Section II gives the general formalism, while section III discusses the dipole sum rule. In section IV we analyse the Compton amplitude, explicitly taking into account pion degrees of freedom: this method has the advantage of dealing with the photoabsorption cross-section rather than the electric dipole one, not directly measurable. Section V enlarges the description to incorporate the $\Delta$ resonance. 


\section{THE MODEL AND ITS NON-RELATIVISTIC LIMIT}

We start with a first quantized relativistic Hamiltonian, which describes the nucleon and pion fields and their interaction through a pseudoscalar coupling $H_{N}+H_{\pi}+H_{N N \pi}$ :

$$
\begin{aligned}
H_{N} & =\int d^{3} x \bar{N}(x)(i \not \partial+M) N(x) \\
H_{\pi} & =\frac{1}{2} \int d^{3} x \vec{\partial} \phi^{i}(x) \vec{\partial} \phi^{i}(x)+\dot{\phi}^{i}(x) \dot{\phi}^{i}(x)+m_{\pi}^{2} \phi^{i}(x) \phi^{i}(x) \\
H_{N N \pi} & =i g \int d^{3} x \bar{N}(x) \gamma_{5} \tau^{i} N(x) \phi^{i}(x)
\end{aligned}
$$

where $m_{\pi}$ is the pion mass and $g$ the $\pi N N$ coupling constant. This Hamiltonian gives rise to the following equation of motion:

$$
\begin{gathered}
(i \not \partial-M) N(x)=i g \gamma_{5} \tau^{i} \phi^{i}(x) N(x) \\
\left(\partial_{\mu}^{2}-m_{\pi}^{2}\right) \phi^{i}(x)=-i g \bar{N}(x) \gamma_{5} \tau^{i} N(x)
\end{gathered}
$$

The antinucleon contribution is small in the low-energy domain, $O\left(1 / M^{2}\right)$, and it can be neglected. The charge density operator then reduces to the sum of the nucleon and pion charge density:

$$
\begin{aligned}
j_{0}(x) & =\rho_{N}(x)+\rho_{\pi}(x) \\
& =e\left[\psi^{+}(x) \frac{\left(1+\tau_{3}\right)}{2} \psi(x)+\dot{\phi}^{2}(x) \phi^{1}(x)-\dot{\phi}^{1}(x) \phi^{2}(x)\right]
\end{aligned}
$$

with the substitution of the field $N(x)$ by the two component field $\psi(x)$. The Siegert theorem guarantees that the pion contribution is a correction of order $1 / M^{2}$ and it can thus be ignored in the charge density. The situation differs if we consider the time derivative of the charge density operator. Using the equation of motion for the time derivatives of the nucleon and pion fields, we get:

$$
\begin{aligned}
\dot{j}_{0}(x) & =e\left[\frac{i}{2 M} \vec{\partial}\left(\psi^{+}(x) \frac{\left(1+\tau_{3}\right)}{2} \vec{\partial} \psi(x)\right)+\vec{\partial}\left(\vec{\partial} \phi^{2}(x) \phi^{1}(x)-\vec{\partial} \phi^{1}(x) \phi^{2}(x)\right)+\right. \\
& \left.+\frac{i g}{2 M} \vec{\partial}\left(\psi^{+}(x) \vec{\sigma}\left(\phi^{2}(x) \tau^{1}-\phi^{1}(x) \tau^{2}\right) \psi(x)\right)\right]
\end{aligned}
$$


Notice that the result is the divergence of a vector, as should be, since the continuity equation is automatically implemented in the theory.

Finally we evaluate the commutator of $j_{0}(x)$ with $\dot{j}_{0}(x)$, ignoring the $1 / M^{2}$ contributions. This commutator plays an essential role in the dipole sum rule and in the definition of the seagull terms in the Compton amplitude. After a cumbersome but straightforward calculation we get the result of Ref. [4]:

$$
\begin{aligned}
{\left[j_{0}(\mathbf{x}, t), \dot{j}_{0}\left(\mathbf{y}, t^{\prime}\right)\right]_{t=t^{\prime}} } & =e^{2}\left[\delta ^ { 3 } ( \mathbf { x } - \mathbf { y } ) \left[\frac{i}{M} \Delta\left(\psi^{+}(x) \frac{\left.1+\tau_{3}\right)}{2} \psi(x)\right)+\right.\right. \\
& \left.\left.+i \Delta\left(\phi^{1}(x) \phi^{1}(x)+\phi^{2}(x) \phi^{2}(x)\right)\right]\right]
\end{aligned}
$$

In Eq. (5) terms with a vanishing expectation value have been discarded. Notice that the explicit inclusion of the pion in the definition of the electromagnetic current replaces the usual seagull term given by the exchange potential $e^{2} \Delta\left(\psi^{+}(x) \psi(x) V_{e x c h}\left(x-x^{\prime}\right) \psi^{+}\left(x^{\prime}\right) \psi\left(x^{\prime}\right)\right)$, by a quantity related to the square of the charged pion field. In the potential case and in the OPEP approximation, the seagull term is represented by the four Feynman diagrams of Fig. V.

In the present description, the intermediate excited states with one pion can be reached by the current and are excluded from the seagull term. In other words the diagrams which can be cut on a pion line between the two photons get an imaginary part. This is the case for the two-body diagrams a,b,c of Fig. V. Thus they do not appear in our new description of the seagull term and only the diagram $1 \mathrm{~d}$ enters.

\section{THE DIPOLE SUM RULE}

The commutator of Eq. (5) enters in the electric dipole sum rule:

$$
\mathbf{S} 1=\frac{1}{2 \pi^{2}} \int_{0}^{\infty} \sigma^{E 1}(\omega) d \omega=\frac{1}{3}\langle\mathbf{0}|[[\mathbf{D}, H], \mathbf{D}]| \mathbf{0}\rangle
$$


where $\sigma^{E 1}(\omega)$ is the electric dipole part of the photoabsorption cross-section and the dipole operator is:

$$
\mathbf{D}=\int \mathbf{x} \cdot j_{0}(x) d^{3} x
$$

The mean value on the ground state has to be evaluated in the centre-of-mass frame. From Eqs. (5) and (6) we get

$$
\frac{1}{2 \pi^{2}} \int_{0}^{\infty} \sigma^{E 1}(\omega) d \omega=\frac{e^{2}}{M} \frac{Z N}{A}+e^{2}\left\langle 0\left|\phi^{\alpha}(0) \phi^{\alpha}(0)-\phi^{3}(0) \phi^{3}(0)\right| 0\right\rangle
$$

The nuclear pion field that enters in the expectation value is the total one in the nucleus. Its square has terms where the pion field originates and is absorbed by the same nucleon. This does not represent a correlation term. In the photoproduction cross-section as well there is a contribution coming from free nucleons. It is then natural to subtract this contribution so as to retain only the exchange pieces, as in the original Bethe-Levinger sum. The new sum rule is written:

$$
\frac{1}{2 \pi^{2}} \int_{0}^{\infty} d \omega\left[\sigma_{A}^{E 1}(\omega)-A \sigma_{N}^{E 1}(\omega)\right]=e^{2} \frac{Z N}{A M}\left(1+K_{4}\right)
$$

where

$$
K_{4}=e^{2}\left[\left\langle 0\left|\phi^{\alpha}(0) \phi^{\alpha}(0)\right| 0\right\rangle-\left\langle 0\left|\phi_{i}^{\alpha}(0) \phi_{i}^{\alpha}(0)\right| 0\right\rangle-(\alpha \rightarrow 3)\right]
$$

The second piece in the brackets represents the contribution of the free nucleons. We have used in Eq. (9) the same notation $K_{4}$ as in our previous paper [3] on the Compton amplitude. This term, which is a genuine seagull term, represents the Thomson amplitude of the charged exchanged pions as given in graph of Fig. Vd. The sum rule Eq. (9) is a new one in which the meson exchange correction on the Compton amplitude is linked to the expectation value of the squared charged pion field.

On the left-hand side of Eq. (9) the cross-section has to incorporate the contributions of all excitations that can be reached with our electromagnetic current. In addition to purely nucleonic states, they include in particular states with pions, arising from the photoproduction process. The corresponding cross-section difference thus extends above the pion 
threshold including also the photoproduction region. It represents the modifications of the E1 cross-section for a nucleon imbedded in the nuclear medium. This includes the giant dipole part and the quasi-deuteron one, as for the usual enhancement factor, but also the changes of the E1 photoproduction cross-section, as due for instance to a Pauli blocking. Accordingly our enhancement factor $K_{4}$ is different from the usual one, $K$. The inclusion

of pion degrees of freedom in the electromagnetic current eliminates from $K$ the terms a, b, c, of Fig. V characterized by a pion propagation between the two photons, which appear as seagulls only in the potential theory. In a world with only nucleons and pions it would thus be possible to evaluate the expectation value of $\phi^{\alpha}(x) \phi^{\alpha}(x)$, from the measured photoabsorption cross-sections.

\section{DISPERSION RELATIONS AND COMPTON AMPLITUDE}

We now turn to the approach in terms of the forward Compton amplitude $f(\omega)$. At zero energy it takes the Thomson values, which for the nucleus, proton and neutron are, respectively:

$$
f_{A}(0)=-\frac{e^{2} Z^{2}}{A M}, \quad f_{p}(0)=-\frac{e^{2}}{M}, \quad f_{n}(0)=0 .
$$

In the high-energy limit it is given by a seagull term $S$, which can be obtained by gauge and covariance constraints [1]:

$$
\begin{aligned}
\left(\varepsilon \varepsilon^{\prime}\right) S & =\varepsilon_{\mu} \varepsilon_{\nu}^{\prime} S_{\mu \nu} \\
\delta^{4}(x-y) \partial_{\mu} \partial_{\nu} S_{\mu \nu} & =\delta\left(t-t^{\prime}\right)\left\langle 0\left|\left[j_{0}(x),\left[j_{0}(y), H\right]\right]\right| 0\right\rangle
\end{aligned}
$$

On the other hand the energy dependence of the Compton amplitude is governed by the time-ordered product given by

$$
T(\omega)=\sum_{n} \frac{\left|\left\langle 0\left|\mathbf{j}(x) e^{i k x}\right| n\right\rangle\right|^{2}}{E_{n}-\omega+\imath \varepsilon}-\frac{\left|\left\langle 0\left|\mathbf{j}(x) e^{i k x}\right| n\right\rangle\right|^{2}}{E_{n}+\omega+\imath \varepsilon}
$$

One can see from Eq. (13) that $T(\omega)$ goes to zero when $\omega$ is well above the excitation energies in consideration: $\omega>E_{n}$. In this case $T(\omega)$ obeys an unsubtracted dispersion relation so that: 


$$
T(0)=\frac{1}{2 \pi^{2}} \int_{0}^{\infty} \sigma\left(\omega^{\prime}\right) d \omega^{\prime}
$$

In contradistinction the dispersion relation for the amplitude $f(\omega)$ requires a subtraction as $f(\omega \rightarrow \infty)=S$. Writing

$$
f(\omega)=T(\omega)+S
$$

we obtain at zero energy

$$
f(0)=T(0)+S,
$$

where $f(0)$ is the Thomson value, $T(0)$ is known from the cross-section integral Eq. (14). Therefore Eq. (16) can be used to determine the seagull term $S$ through a sum rule. As in the previous section we have to subtract from $f(\omega)$ the free nucleon part so as to retain only the exchange part, leaving only the term of diagram 1e, which we denote by $S_{4}$ :

$$
S_{4}=-e^{2} \frac{Z N}{A M} K_{4}
$$

Our sum rule is written:

$$
\frac{1}{2 \pi^{2}} \int_{0}^{\infty} d \omega\left[\sigma_{A}(\omega)-A \sigma_{N}(\omega)\right]=e^{2} \frac{Z N}{A M}\left(1+K_{4}\right)
$$

We find a relation similar to that given in Eq. (9), but the full cross-section rather than the electric dipole one is involved.

It is clear from Eq. (5) that the seagull term depends on the definition of the electromagnetic current. In the present work we do not want to incorporate the full complexity of this current. Our scope is limited: we consider only nucleonic and pionic degrees of freedom. In this way we obtain a simple expression for the seagull in terms of the pion field. The same remark applies to the time-ordered product. Accordingly, in our sum rule, such effects as shadowing of the photoabsorption cross-section due to vector dominance are left out of our considerations. This is why a cut-off in energy has to be applied in the integral over the cross-section. 
We now define this cut-off, denoted by $\infty$ in the integral over the cross-section of Eq. (14). This maximum energy depends on the model that we use. In the original Bethe-Levinger description, the electromagnetic current does not create pions. The intermediate states $|n\rangle$ in the expression of $T(\omega)$ in Eq. (13) are thus purely nuclear. Large $\omega$ values mean well above the nuclear excitation energy, in such a way that $T(\omega) \rightarrow 0$. In this case an energy in the vicinity of the pion mass can be considered large and the integral of the cross-section is restricted to the energy below the pion threshold. In the present description the potential approach is given up. Mesons are now explicitly taken into account, we retain only the pion and we ignore the nucleonic excitations. High energy, in this case, means above the photoproduction region (in the absence of nucleonic excitations), $\omega \approx$ few hundred $\mathrm{MeV}$ is considered large. Thus the cross-section integral should be cut at $\omega_{\max } \approx 300-500 \mathrm{MeV}$.

Let us elaborate better on the choice of the cut-off, which is a crucial point in our work. Our description of the nucleus is restricted to a certain type of excitations: purely nuclear in the original Bethe-Levinger sum rule, nuclear plus pionic in the present case. It is then necessary to reach a consistent description with only those excitations and to eliminate the other ones, in particular from the cross-section, which impose a cut-off in the integral. The idea is that there are well separated energy domains, where only certain excitations dominate: only nuclear at low energies $\omega_{\max } \leq m_{\pi}$; nuclear and pionic in the region up to $\omega_{\pi} \approx 300-500 \mathrm{MeV}$. This is only approximate. For instance the nuclear excitations extend also above the pion threshold, and this is a well-known problem for the determination of $K$. Similarly we will see that higher excitations such as $\Delta$ mix in the photoproduction region and we will have to extend the description to incorporate the $\Delta$. However, for the moment we ignore this difficulty. In order to reach a consistent theory with a chosen set of excitations, the maximum energy should be large enough for $T\left(\omega_{\max }\right)$ to vanish and at the same time small enough for the other excitations not to show up in the cross-section. The expectation value of the squared pion field, which builds the seagull term $K_{4}$ is an important quantity. We follow here the ideas of Chanfray and Ericson [5] who have shown that it governs the restoration of chiral symmetry in nuclei. It also enters as a pion exchange 
contribution to the $s$-wave pion optical potential. In Ref. [5] it is expressed in terms of spinisospin response function. If the only hadrons present in the nucleus are nucleons, the static approximation can be used. It leads to the following equation:

$$
\frac{Z N}{A M} K_{4}=\frac{g^{2}}{4 M^{2}} \int \frac{d^{3} q}{(2 \pi)^{3}} \sum_{i \neq j} \frac{\left\langle 0\left|\left(\vec{\sigma}_{i} \vec{q}\right)\left(\vec{\sigma}_{j} \vec{q}\right) \tau_{i}^{\alpha} \tau_{j}^{\alpha}\right| 0\right\rangle}{\left(q^{2}+m_{\pi}^{2}\right)^{2}}
$$

This quantity involves longitudinal spin-isospin correlations and it is related to the pion excess number $[3]$.

The theoretical evaluation of $K_{4}$ with the nuclear matter spin-isospin correlations of Ref. [6], gives a value $K_{4}=0.16$, which is much smaller than the usual factor $K$, in agreement with Noble's findings [4]. This is due to a nearly complete cancellation between the scalar and the tensor pieces of $K_{4}: K_{4 \text { scalar }}=-0.68, K_{4 \text { tensor }}=+0.84$. The scalar part has essentially the same value as in the free Fermi gas model. It represents the Pauli blocking effect for the contribution of Fig. 2a to the Compton amplitude. On the contrary, $K_{4}$ tensor vanishes in a free Fermi gas and arises only from tensor correlations, which are mostly responsible for the quasi-deuteron cross-section (see diagrams of Figs. $2 \mathrm{~b}$ and $2 \mathrm{c}$ ). In our analysis a small $K_{4}$ value does not contradict the sum rule in Eq. (18). It implies that the cross-section integral exceeding the classical sum rule should also be small. As a consequence the integral of the quasi-deuteron cross-section should be nearly equal to the Pauli quenching of the photoproduction one.

Let us now turn to the analysis of the experimental data and discuss whether $K_{4}$ is experimentally accessible. The present discussion incorporates only nucleons and pions. This is not realistic as the $\Delta$ resonance strongly influences the photoabsorption nuclear and nucleonic cross-sections. The question is then whether this influence prevents an experimental determination of $K_{4}$. Let us recall that the $\Delta$ is not appreciably modified in the nucleus: its influence, which is overwhelming in the separate cross-section individually, is therefore less dominant in their difference. It is thus conceivable that, by introducing a suitable cut-off in the integral over the cross-section difference, we may limit this influence, at the same time preserving the information on the nucleon-pion sector. 
However this is not possible because the Pauli blocking itself is affected by the existence of the $\Delta$. It is indeed known that the modification of the pion propagation in the nuclear medium, due to the virtual $\Delta$-hole excitations, makes the Pauli blocking less effective [7]. It is thus not possible to disentangle the $\Delta$ in the pion photoproduction region. Therefore the description should not be restricted to nucleons and pions, but also incorporate the $\Delta$ resonance, which is done in the next section. This is not a disadvantage. Anyhow a determination of $\left\langle\phi^{2}\right\rangle$ that would not incorporate the $\Delta$ would not be realistic, since the $\Delta$ is so strongly excited by the pion degree of freedom.

\section{THE $\triangle$ EXCITATION}

We now extend this description to incorporate the $\Delta$ excitation, which we treat as a stable particle. This inclusion changes again both $T(0)$ and $K_{4}$. This last quantity, which is the Thomson amplitude on the exchanged pions, has to take also the $\Delta$ into account as given by the graphs of Fig. $3 \mathrm{~b}$. We have kept the diagrams with one $\Delta$. The many-body graphs corresponding to Fig. 3a and $\mathrm{b}$ are shown in Fig. 2.

In the dispersion relation for $T(0)$ the integral over the cross-section has to include also the region of $\Delta$ and $\Delta \pi$ production, i.e. the region up to $\omega_{\max } \approx 700-800 \mathrm{MeV}$. This region covers the near-threshold one for the $\Delta \pi$ excitations, which is expected to experience a strong medium modification. Indeed, in the nuclear medium, the elementary process $\gamma+N \rightarrow \Delta+\pi$ should give rise, below threshold, to a quasi-deuteron with a $\Delta$, absent for free nucleons. The relation (18) remains true with the new definition of $K_{4}$ and the new value of the maximum energy in the integral.

The seagull term of Fig. 2 can be concisely expressed, as before, as the expectation value of the square pion field, Eq. (10). This expression was obtained previously without $\Delta$ but it remains valid also in this more general case. Actually the expectation value is changed by the introduction of the $\Delta$, but not the operator form appearing in Eq. (5). For the actual evaluation of $K_{4}$, the $\Delta$ excitation shoud be incorporated in the nuclear response. In 
the static approximation the correlations with $\Delta$ excitation are not available. However an estimate $K_{4}$ can be obtained in the following way. The spin-isospin correlations also enter in the pion excess number, which is given by a similar expression, with a different power of $\omega_{q}$ in the denominator $\left(\omega_{q}^{3}\right.$ instead of $\left.\omega_{q}^{4}\right)$. This is reflected in the following relation [3] between the seagull term and the pion excess number per nucleon, $n_{\pi}$ :

$$
K_{4}=\frac{16}{3} n_{\pi} m_{\pi}\left\langle\frac{1}{\omega_{q}}\right\rangle
$$

where $\left\langle 1 / \omega_{q}\right\rangle=\left\langle\left(q^{2}+m_{\pi}^{2}\right)^{-1}\right\rangle$ is an average over the pion momentum. This relation, which holds as well with the $\Delta$, can be used to estimate $K_{4}$. The pion excess has been evaluated by Freeman et al. [9] who pointed out the important role of $\Delta$ in the production of the pion excess. In nuclear matter we deduce from their work the $\Delta$ contribution to the excess

$$
n_{\pi}^{\Delta} \approx 0.18-0.04 \approx 0.14
$$

where the first number in the difference is the total value and the second the purely nucleon one. For the average pion energy, we take that of the tensor, which dominates the $\Delta$ contribution $\left\langle 1 / \omega_{q}\right\rangle \approx(550 \mathrm{MeV})^{-1}$. From Eqs. (20) and (21) we estimate for the seagull term $K_{4}^{\Delta}$ of Fig. $2 \mathrm{c}: K_{4}^{\Delta}=1.3$. On the other hand, for the nucleon contribution $K_{4}^{N}$ of Fig. $2 \mathrm{a}$ and $2 \mathrm{~b}$, the explicit evaluation with the nuclear matter correlations of Ref. [6] gives a small value $K_{4}^{N}=0.16$ as already mentioned. The overall theoretical prediction is then

$$
K_{4}=K_{4}^{N}+K_{4}^{\Delta} \approx 1.5
$$

Let us now turn to the experimental value, deduced from the relation (18) and the experimental cross-sections [10], [11]. The cross-section per nucleon is shown in Fig. 4 for uranium together with the nucleon one and the difference. Notice the sizeable excess of the nuclear cross-section over the free one in the region of $400-500 \mathrm{MeV}$, as expected from the quasi-deuteron with $\Delta$ mechanism. An evaluation of the cross-section integral of Eq. (18) depends somewhat on the exact value of the cut-off. We have taken three choices: $\omega_{\max }=700,800$ and $1000 \mathrm{MeV}$. We have used the data of Ref. [10] up to $100 \mathrm{MeV}$ and 
those of [11] above. We obtain, for the value of $K_{4}: \approx 1.5,1.3,1.1$, respectively for $\omega_{\max }=$ $700,800,1000 \mathrm{MeV}$, with an error of about $10 \%$. At this stage, we would like to comment our value of $K_{4}$, which is in reasonable agreement with the theoretical estimate of Eq. (22). We find nearly the same result as in the classical Bethe-Levinger sum rule [2], in spite of the explicit inclusion of extra degrees of freedom, the $\pi$ and $\Delta$. This is understandable as the inclusion of the pions only brings the value of $K$ down to practically zero (linked to the Pauli blocking of the photoproduction cross-section). The value of $K$ is then restored by adding the $\Delta$ degrees of freedom.

Not surprisingly, the value of $K_{4}$ displays a certain sensitivity to the cut-off. Its determination is therefore approximate. However, this limited information is interesting because even the sign of $K_{4}$ is not a priori obvious. We find it to be positive, which establishes the sign of correlation part of the expectation value of $\phi^{2}$. This result is quite compatible with the existence of a pion excess in nuclei, of the predicted magnitude. It also confirms the important role played by the $\Delta$ resonance in this excess.

The question that can be raised is whether $\phi^{2}$ is saturated by incorporating in the description only nucleons and $\Delta$, which fixed the cut-off in the region of $800 \mathrm{MeV}$. In principle the answer is no, other resonances which decay via the pion emission could contribute as well. However the inclusion of nucleons and $\Delta$ only to saturate the pionic degrees of freedom is a common procedure and it should give the bulk of the effect.

We now address the question of consequences for chiral symmetry restoration in nuclei and of the pion exchange correction in $\pi$-nucleus scattering. A positive value of $K_{4}$ means an acceleration of the restoration process [5] and a repulsive exchange contribution in the $s$-wave optical potential. Using the relation between $K_{4}$ and $\Delta \Sigma_{\text {pion exch }}$ given in Ref. [5], we deduce from the value $K_{4} \approx 1.2$ in uranium, a nucleus that we consider representative of nuclear matter, $\Delta \Sigma_{\text {pion exch }}\left(\rho_{0}\right) \approx+4.5 \mathrm{MeV}$ (as compared with $\Sigma_{N} \approx 45 \mathrm{MeV}$ ), an acceleration of the restoration process by $10 \%$.

On the other hand the pion exchange contribution to the $s$-wave optical potential parametrized as $2 m_{\pi} V_{o p t}=-4 \pi \rho b_{0}^{e f f}(\rho)$ is 


$$
b_{0 \text { exch }}^{\text {eff }}\left(\rho_{0}\right)=-\frac{\Sigma_{\text {pion exch }}}{4 \pi f_{\pi}^{2}\left(1+\frac{m_{\pi}}{M}\right)}=-0.004 m_{\pi}{ }^{-1} .
$$

We recall that the experimental value from the pionic data, i.e. at a density somewhat lower than the normal one, is $b_{0}^{e f f}\left(\rho \approx 0.7 \rho_{0}\right) \approx-0.03 m \pi^{-1}$. Scaling the calculated exchange contribution linearly in the density, we find that pion scattering off exchanged pions provides about $10 \%$ of the measured repulsive potential.

In conclusion, we have established a new sum rule, which is an extension of the BetheLevinger one, and which includes also the pion and $\Delta$ degrees of freedom. Our sum rule links the seagull term of the Compton amplitude, i.e. the Thomson scattering on the exchanged pions, to the integral of the cross-section that covers the region of nucleon, pionic and $\Delta$ excitations, i.e. up to $\approx 800 \mathrm{MeV}$. As the seagull term is linked to the expectation value of the pion field squared (correlation piece), the experimental value that we derive from the sum rule establishes the positiveness of this quantity, consistent with the existence of a pion excess in the nucleus. It also indicates a slight acceleration of chiral symmetry restoration in nuclei, due to the interaction and a repulsive pion-exchange contribution to the $s$-wave optical potential. 


\section{REFERENCES}

[1] P. Christillin and M. Rosa-Clot, Nuovo Cimento A 28 (1975) 29.

[2] J.S. Levinger and H.A.Bethe, Phys. Rev. 78 (1950) 115.

[3] M. Ericson and M. Rosa-Clot, Phys. Lett. B188 (1987) 11.

[4] J.V. Noble, Phys. Rep. 40 (1978) 241.

[5] G. Chanfray and M. Ericson, Nucl. Phys. A556 (1993) 427.

[6] S. Fantoni and S. Rosati, Nuovo Cimento A 43 (1978) 413.

[7] W.M. Mac Donald, E.T. Dressler and J.S. O'Connel, Phys. Rev. C10 (1979) 455.

[8] M. Ericson, FestiVal: Festschrift for Valentine Telegdi, ed. K. Winter, p. 65.

[9] B.L. Freeman, V.R. Pandharipande and R.B. Wiringa, Phys. Rev. Lett. 51 (1983) 763.

[10] A. Lepretre, H. Beil, R.Bergère, P. Carlos, J. Fagot, A. Miniac, A. de Veyssière, Nucl. Phys. A367 (1981) 237.

[11] Th. Frommhold, Phys. Lett. 295B (1992) 28; U. Kneissl, private communication; M. Aghinolfi et al., Phys. Rev. C47 (1993) R922; G. Gervino, private communication. 


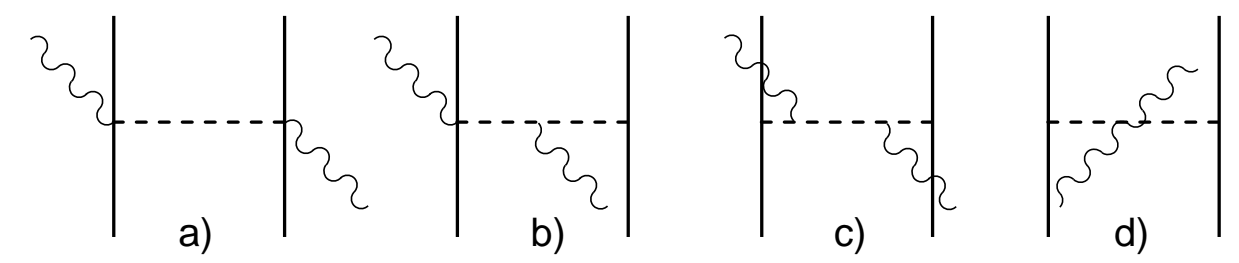

Figure 1: Seagull contributions in the OPE potential model.

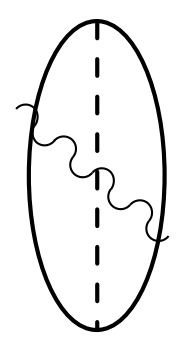

a)

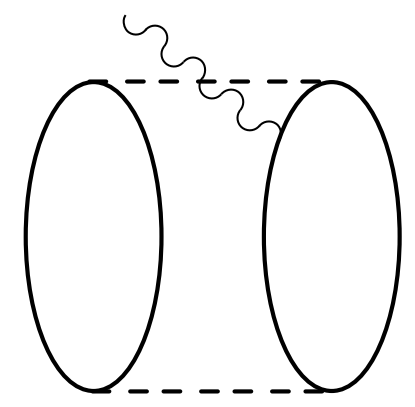

b)

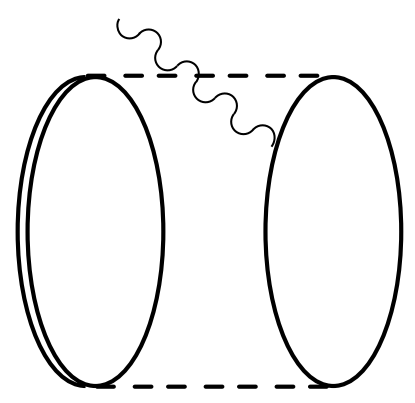

c)

Figure 2: Many-body diagrams for the pion-exchange seagull contribution to the Compton amplitude. 


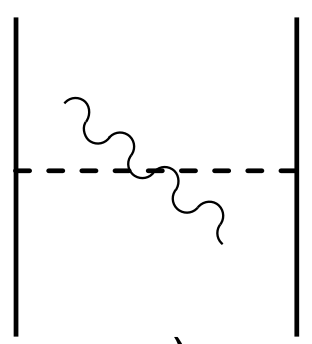

a)

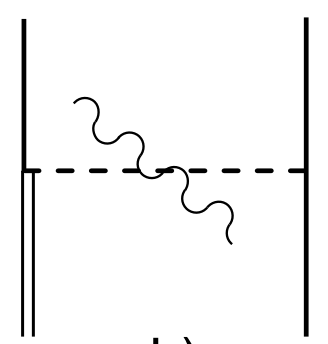

b)

Figure 3: Seagull contributions when $\pi$ and $\Delta$ degrees of freedom are explicitly taken into account. 


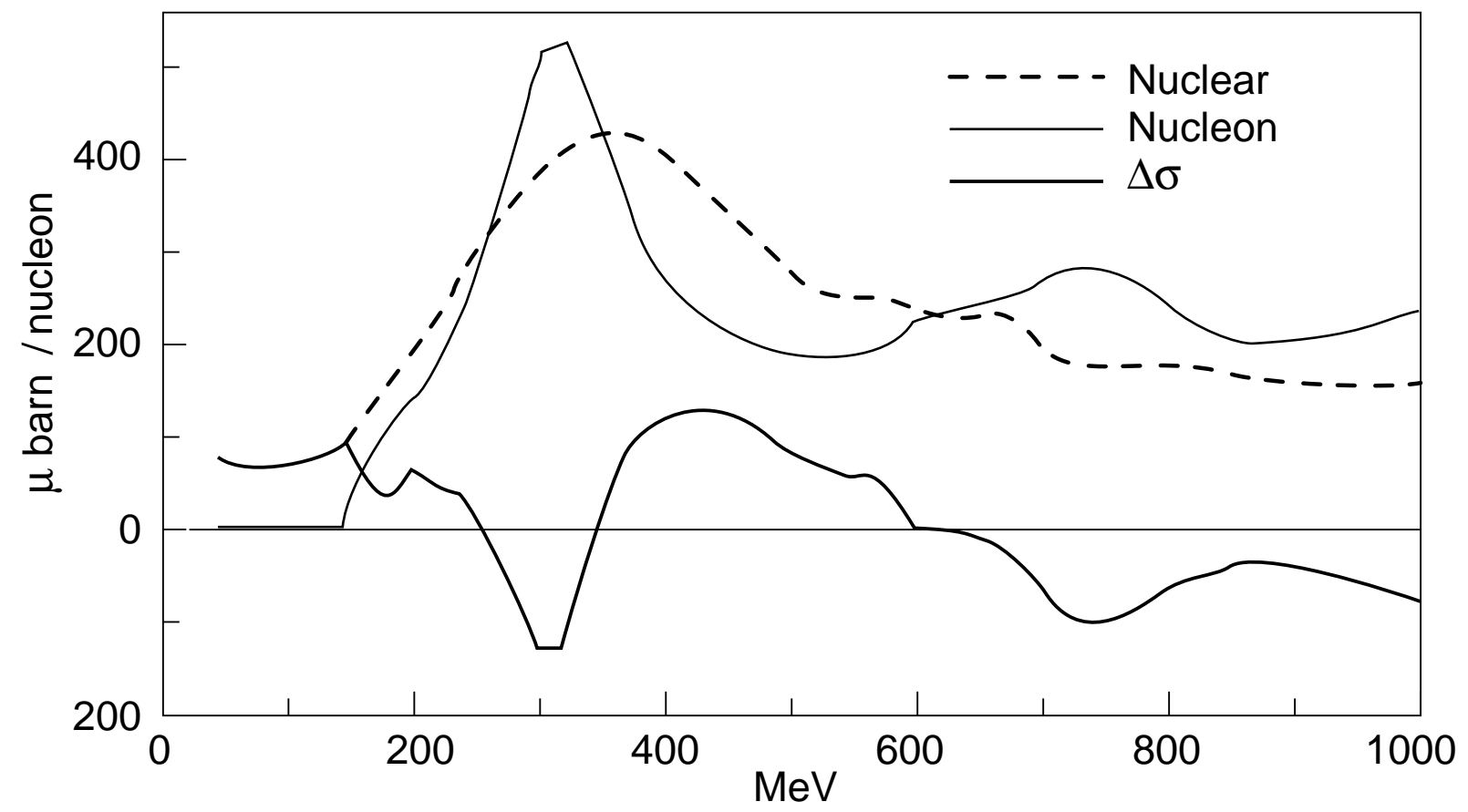

Figure 4: Plot of the cross-section per nucleon in uranium (nuclear), of the weighted average of the proton and neutron cross-sections (nucleon) and of their difference $(\Delta \sigma)$. 